\title{
Cataract Surgery Outcome in Relation to Posterior Capsular Rupture; A Retrospective Study
}

\author{
Dr. Ali A Taqi Al-Saffar ${ }^{1 *}$, Dr. Shilan Salih Hama² \\ ${ }^{1}$ Assistant Professor, School of Medicine, University of Sulaimani, Kurdistan-Iraq \\ ${ }^{2}$ Ophthalmologist, Shaheed Aso Eye hospital, Iraq
}

\author{
DOI: $10.36348 /$ simps.2020.v06i08.009 \\ | Received: 16.07.2020 | Accepted: 24.07.2020 | Published: 29.08.2020 \\ *Corresponding author: Dr. Ali A Taqi Al-Saffar
}

\section{Abstract}

Background: The Posterior capsule rupture is one of the most serious intra-operative cataract surgery complication that affect the success of Cataract operation and its outcome, results in relation to visual outcome and the quality of life to the patients. Objectives: 1) To identify our rate of Posterior capsule rupture. 2) To figure out possible related risk factors. Type of study: A retrospective study of all Cataract surgeries done in 2016 in Shaheed Dr. Aso teaching Eye specialty hospital, which is the only and main eye hospital in Sulaimaniya city, Kurdistan region of Iraq. Statistical Analysis: Statistical Package of Social Sciences 24 (SPSS-24). Patients and Methods: A retrospective study done on the file data of 1057 files(eyes/patients) underwent cataract surgeries either by phacoemulsification, extra-capsular cataract extraction or small incision cataract surgery at 2016, all useful information and details from the files were taken and analyzed. Inclusion Criteria: All good documentation files of cataract surgeries taken, regardless of cataract maturity classification as immature, mature and hypermature and regardless of surgeries done by hands of specialist, or training surgeons under supervision of seniors and regardless of types of machines of phacoemulsification, 1057 files taken. Exclusion Criteria: Vague or defective and in adequate information and documentation files were excluded, we exclude eight files only. Results: The rate of Posterior Capsular Rupture was 58 (5.4\%) eyes in all cataract surgeries, 1057 eyes, that done by hands of highly qualified specialists or supervising the training resident surgeons, of those 58 eyes, 32 eyes ends with primary intraocular lens implantation, 16 eyes with secondary intraocular lens implantation and 10 eyes left as Aphakia. Risk stratification done by data available preoperatively, the maturity of cataract in which hypermature cataract had (22.2\%), (95\% CI 1.17 to 16.98; OR: 5.40) and P>0.001 risk for Posterior capsular rupture, and type of surgery in which ECCE had $6.9 \%$ risk factor (95\% CI 1.03 to 3.13; OR 1.80), P> 0.036. Conclusion: Although Posterior capsular rupture in the average but it's still significant.

Keywords: Extra capsular cataract (ECCE), Phacoemulsification, Manual small incision cataract surgery (SICS), Posterior capsular rupture (PCR), Glaucoma, Pseudoexfoliation syndrome (PXS).

Copyright @ 2020: This is an open-access article distributed under the terms of the Creative Commons Attribution license which permits unrestricted use, distribution, and reproduction in any medium for non-commercial use (NonCommercial, or CC-BY-NC) provided the original author and source are credited.

\section{INTRODUCTION}

The most serious intra- operative Cataract surgery complication is rupture of the posterior lens capsule (PCR), and may related to poor visual outcome, eye with the PCR were 3.8 time more likely to have best corrected visual acuity less than 6/12 [17].

Cataract is the leading cause of preventable blindness in the world, whereas the most effective surgical procedure in all of medicine is cataract extraction with intraocular lens (IOL) implantation [1]. A cataract is the opacity of the lens of the eye that causes partial or total blindness. In ancient times, cataract was not recognized as opacification of the crystalline lens, rather it was thought to be a suffusion forming between the pupil and the lens. The term cataract therefore arose after translation of the Arabic term for 'suffusion' into Latin cataracta (meaning waterfall or obstruction of flow), similar to the cataracts that impeded the navigation of the Nile river [2].

The number of blind due to cataract in the world is not accurately known, but it has been estimated at various times by World health organization (WHO), thus, it was reported that there might be $10-15$ million blind globally in 1972 [4].

In 2002, the WHO estimated that cataract caused reversible blindness in more than 17 million $(47.8 \%)$ of the 37 million blind individuals worldwide, 
and this number is projected to reach 40 million by 2020, when a WHO Study Group on the Prevention of Blindness was convened, this value was recognized to be an underestimate, even though based on information provided by Member States [1].

The current rate of cataract surgery in the USA is 5000 per million people per year, but in Africa the average rate is only about 200 per million people [4]. In the USA in the late 1990s, 1.35 million cataract operation were done per year at a cost of US\$3.4 billion [1], however, the cost of not treating cataracts that is, the cost of nonfunctional vision secondary to cataract is much greater because of the loss of jobs and need for care $[7,1]$.

The most common cause of Cataract is the age relate, the etiology of age-related changes in the lens is not fully understood and is likely to be multifactorial [1, 10]. There are three main types of age-related cataracts defined by their anatomical appearance, nuclear, cortical, and posterior subcapsular. They can present alone or in combination. Typically changes are bilateral, but they are commonly asymmetrical [11].

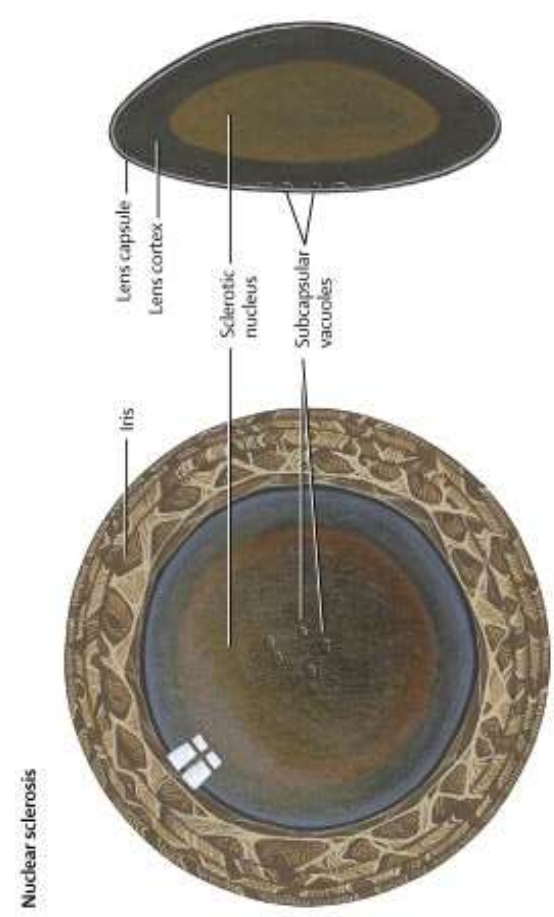

\section{Cataract Removal Surgical Procedures Extra capsular cataract extraction (ECCE)}

The extra capsular procedure involves careful removal of the anterior lens capsule, delivery of the lens nucleus through an incision at the junction of the cornea and sclera, and aspiration of the residual cortical lens material. This procedure leaves the posterior lens capsule intact along with the equatorial zonular attachments - the capsular bag. The preservation of the capsular bag facilitates better anatomical location of an implanted intraocular lens $[1,2]$.

\section{Phacoemulsification}

Phacoemulsification, first developed by Kelman in 1967, is currently the procedure of choice for the surgical management of cataracts $[13,15]$. It can be viewed as a modified form of extracapsular cataract extraction. It also means that much of the capsular bag is left intact. The key difference is that, rather than delivering the whole lens nucleus through a large limbal incision (between the cornea and the sclera) about 11 $\mathrm{mm}$ wide, a high-frequency ultrasonic probe is inserted through a smaller corneal incision (about $3 \mathrm{~mm}$ ) and energy is delivered to emulsify the lens nucleus inside the eye. The fragments are subsequently aspirated through the same probe. From the points of view of refraction and postoperative wound care, the smaller the incision, the fewer the complications, such as collapse of the contents of the eye during surgery and sutureinduced astigmatism. One of the challenges of a smaller incision that was initially encountered was that many replacement intraocular lenses exceeded the size of the incision [1]. This problem was overcome by the development of the foldable intraocular lens; it has a diameter of $6 \mathrm{~mm}$ but can be folded and inserted through a $3 \mathrm{~mm}$ incision. Most phacoemulsification incisions today are $3 \mathrm{~mm}$ or less. Our experience has been that smaller incisions have decreased the duration of the procedure and, more importantly, hastened postoperative visual recovery [3].

\section{Manual Small Incision Cataract Surgery (SICS)}

Has become popular in India in the last decade

[21], it is often used as alternative to 
phacoemulsification its ideal for developing countries when expensive instrumentation required with very high volume cataract surgery needed ${ }^{9}$. The considerable handling in the anterior chamber during nucleus delivery increases the chance of iris injury, striate keratitis and posterior capsular rupture [21].

\section{Complication of the Cataract Surgery \\ Posterior Capsular Rupture (PCR) \\ Capsular Anatomy}

Lens capsule is an elastic, clear, basement membrane composed of type 4 collagen produced by lens epithelial cells. The zonular fibers insert on the equatorial lens capsule, anteriorly $1.5 \mathrm{~mm}$ onto the anterior capsule and posteriorly $1.25 \mathrm{~mm}$ on to the posterior capsule [1], the capsule has variable thickness. Although the capsule is thickest (17 to $23 \mu \mathrm{m}$ ) near the anterior and posterior equator where the zonular fibers attach, it is only 2 to $4 \mu \mathrm{m}$ thick at the posterior pole. The anterior capsule is considerably thicker than the posterior pole (14 $\mu \mathrm{m}$ in adults), and it continues to increase in thickness with age [5]. The posterior capsule may particularly fragile in patients with posterior lenticonus, the posterior capsule act as an anatomical barrier between the anterior and posterior chamber, it limit spread of infection and inflammation process [11].

\section{Common Predisposing Factors of Posterior Capsular Rupture \\ Patient Related}

Systemic disease: include diabetes, neurological and mental disorder they may have involuntary movements and affects patients cooperation, systemic medication like alpha blocker lead to floppy iris syndrome during surgery [11].

Extra ocular: includes deep set eye and narrow palpebral apertures lead to difficulties during various steps of surgery, corneal opacities and endothelial dystrophies which associated with poor intraoperative visibility [1].

Intra ocular: both shallow anterior chamber as in angle closure glaucoma and very deep anterior chamber as in high myopia increase risk of PCR, type of cataract also has a role in increasing the risk like hyper mature, intumescent, posterior polar and post traumatic cataract, Pseudoexfoliation will increase the risk it associated with poor dilatation of pupil and zonular dehiscence ${ }^{11,}$ also previous ocular surgery specially vitrectomy with or without silicone oil, Patients who have had a vitrectomy also face a higher risk either due to a capsular defect, if lens touch from the vitrector was the cause of the post- operative cataract, or because of a floppy, less stable posterior capsule during phacoemulsification due to the loss of stabilization from the absence of the vitreous face and intravitreal injection [12].
Surgeon Related: An inexperienced surgeon (e.g. a resident) [5], learning curve and high volume CAMP surgery [11]. Intraoperative: small rhexis with rhexis block, rhexis- radial tear and fluid imbalance [11].

Machine Related: Unfamiliar machine, machine malfunction, microscope issue and Bottle- over [11].

\section{Causes of Capsular Rupture}

The causes of rupture of the posterior capsule usually involve touch of surgical instruments and can be caused at any stage of the cataract removal procedure. Capsular block from excessive hydrodissection can cause a capsular rupture even prior to phacoemulsification. Other more common causes of capsular rupture are touch from the phacoemulsification probe or second instruments during the phacoemulsification of the lens or from irrigation/aspiration instruments during cortical cleanup [12].

The postoperative sequels are associated with size of rent, vitreous loos, nucleus drop, cortex drop or IOL drop.

The decision about the position of IOL placement depends on the size of the posterior capsular defect. If it is a small focal defect, the lens can still be placed carefully in the bag, made safer if a posterior capsulorrhexis is performed [14].

\section{Significance of Posterior Capsular Rupture Recovery Periods}

There are numerous studies quantifies the postoperative recovery difficulties, loss of daily income and extra cost of medication, if IOL implant at posterior bag, the visual outcome recovery uneventful but patients need medications for long period and more symptomatic [11].

\section{Visual Acuity}

PCR associated with poor visual acuity, the visual acuity of $6 / 12$ or better found in $55-67 \%$ of patient with ECCE and PCR, the outcome has improved extremely with advent of phacoemulsification the rate of best visual acuity increased to $84-98 \%$ of $6 / 12$ and better with only few studies have shown no significant visual acuity difference between the cases with and without PCR [11].

\section{Need for the Secondary Surgery}

The most common surgery related to PCR is anterior vitrectomy mostly done at time of operation with IOL implantation, other procedure which may need it are pars plana vitrectomy for nuclear fragmentation, retinal surgery, scleral fixation IOL, any second surgery lead to delay rehabilitation and more cost on patient [11]. 


\section{Complication of PCR}

\section{The Most Frequent Complications Are}

1. Retinal detachment: the most frightful complication of the PCR, after 20 years of cataract surgeries the patient had fourfold risk to develop retinal detachment this risk increase to fivefold with patient had PCR before, the Swedish capsular rupture study group report there is $4 \%$ incidence of retinal detachment after 3 years of PCR comparable to non PCR cataract surgeries group the incidence $0.03 \%$ [11].

2. Cystoid macular edema CME: is a common complication after complicated cataract surgeries the subclinical CME is about $30 \%$, while the clinical CME is about 1-2\%, after PCR and vitreous loss clinical CME reported to be $21 \%$.

3. Endopthalmitis: is a one of the most feared complication of the cataract surgeries due to its devastating consequence, the most common risk factor is PCR [12].

4. Increased IOP: the PCR is a strong risk factor for increased IOP at postoperative days, the incidence of increasing the IOP at first day postoperative is $20 \%$ [11].

5. Persistent corneal edema and posterior dislocated IOL [14].

\section{OBJectives}

To identify the rate and the risk indicators for posterior capsular rupture (PCR) in the Shaheed Dr. Aso teaching Eye Hospital.

\section{Patients And Methods}

This retrospective study which included 1057 files (eyes, patients) conducted in Shaheed dr. Aso eye hospital in Sulaimaniya city in Kurdistan region/Iraq, included cases which were operated between early Jan 2016 to late December 2016, done by ophthalmologists with different surgical experience including specialties, post graduate student and training residents at Shaheed Dr. Aso teaching eye Hospital. Variable demographic data gathered included age, gender and residency. Other information like visual acuity, IOP, laterality of eyes, axial length, related ocular diseases like glaucoma and Pseudoexfoliation.

The presence of hepatitis virus positive test in pre-operative laboratory notes, past medical and social history including smoking and allergy to drugs extracted from the notes recorded by anesthesiologist or physician preoperatively, Presence of comorbidities like hypertension and diabetes was focused on as they are related to incidence of PCR [22].

The type of cataract included nuclear sclerosis, cortical cataract, mature cataract, immature cataract.
The visual acuity has been transformed to decimal values to facilitated statistical analysis, because there was a problem in conversion of small values like counting finger, hand motion and light perception, as a result the decision made to use Grover et al., [8] calculation. The estimated decimal VA of 0.0025 for CF, 0.002 for HM, 0.0016 for "light perception" (LP), and 0.0013 for NLP, were used which would only indicate a difference of 0.1-log-unit step between $\mathrm{CF}$ and HM and between HM and LP, for this purpose.

The procedures adopted for cataract removal included phacoemulsification, ECCE and SICS.

The immediate surgical outcomes were classified according to possibility of posterior capsular rupture/ tear (PCR) as eventful or smooth uneventful operations. The type of IOL used after PCR also taken into consideration.

Exclusion Criterion: Files with defective important information and inadequate documentation of necessary information.

Inclusion Criteria: Files with clear documented information about type and result of operation with patients' general medical condition and ophthalmological finding.

\section{Statistical Methods}

Data collected were documented and analyzed using IBM SPSS Statistics 24 (IBM SPSS Statistics for Windows, IBM).

\section{RESUlTS}

Of the 1057 eyes cataract operations, 58 (5.5\%) eyes had PCR regardless of vitreous state (vitreous loss or not) and regarded as eventful, while uneventful smooth operations were 999 (94.5\%) eyes.

Of the 1057 patients (eyes) were included, age range in years was 1-110 years with the average mean 64.02, median 65.00 and standard deviation 13.406, The percentage of male was 498 cases $(47.1 \%)$ and female was 559 cases $(52.9 \%)$, the residencies of cases participated in this study were different those who were inside Sulaimaniya city and the surroundings.

Regarding the PCR, 58 eyes, nineteen eyes had posterior chamber IOL in same session, thirteen eyes had either AC or Artisan IOL in time of operation, twenty six of them left as aphakia, and from these 26 aphakia patients, 17 of them underwent secondary IOL implantation, 16 eyes were successful either putting AC IOL or Artisan, one of them left aphakia again as no IOL can be implanted., so total AC-IOL/Artisan IOL were 29 eyes and total eyes/patients that left aphakia are 10 (Figure-4). 


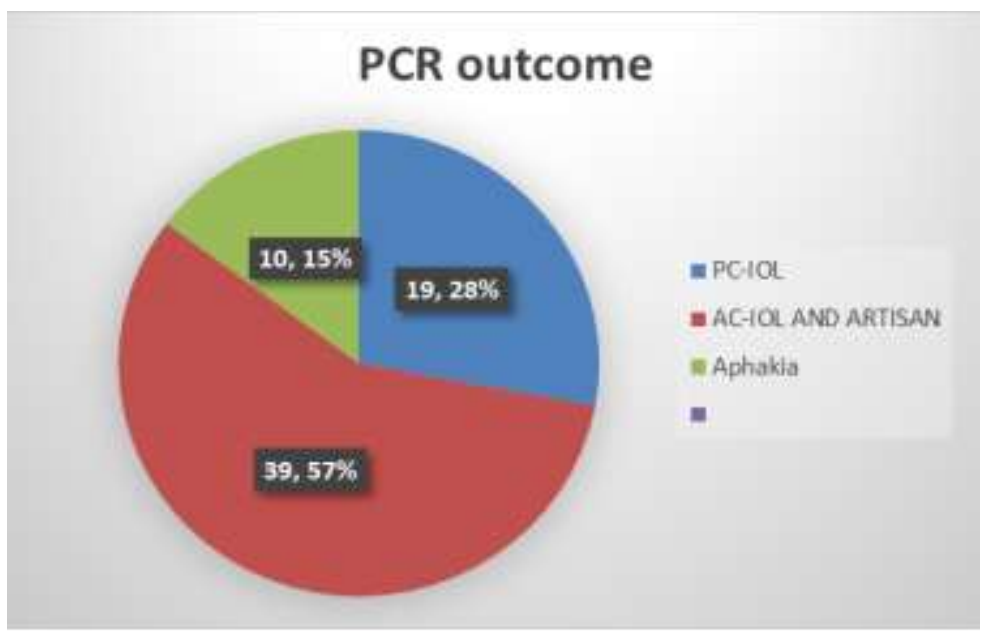

Fig-1: Pie chart of type of IOL implant after PCR

The type of operation, 548 eyes $(51.6 \%)$ ECCE, 471 eyes $(44.6 \%)$ of phacoemulsification, 35 eyes $(3.3 \%)$ of SICS, we have 3 missing case in which the type of operation was not written and all of them had PCR and left as aphakia (Figure-3).

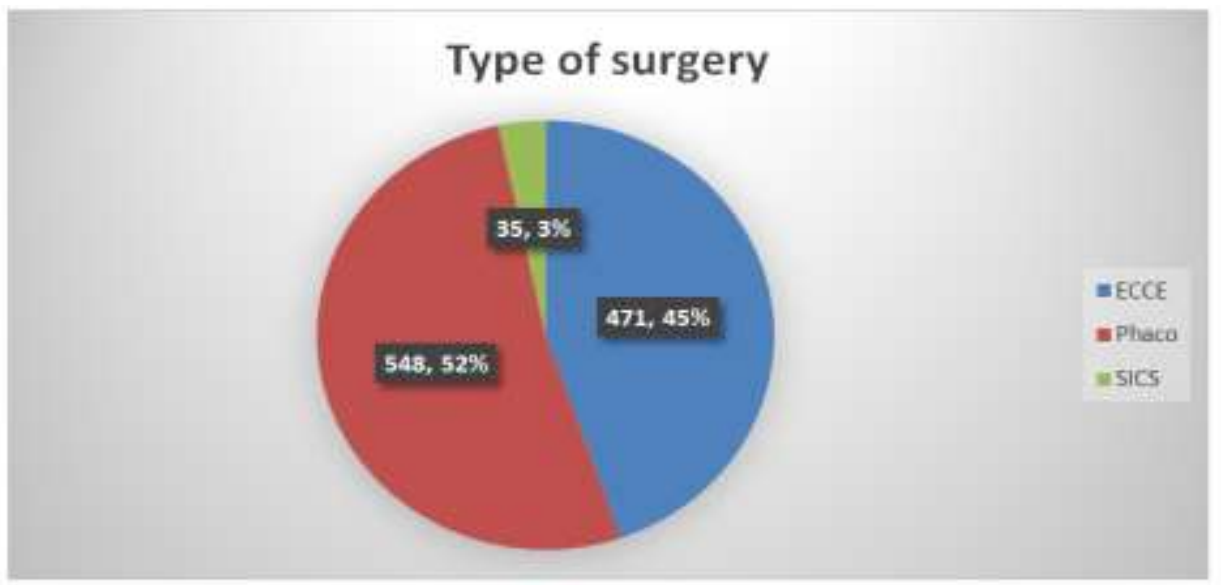

Fig-2: The pie chart for types of cataract operation.

Regarding overall cataract surgical outcome in relation to correction of refractive status with either primary PC-IOL or foldable IOL 999 eyes (good prognosis anticipated), eyes with PCR as follows, 19 eyes with primary PC-IOL, 29 eyes ends with primary or secondary AC-IOL or Artisan iris fixated IOL (fair prognosis anticipated), while 10 eyes unfortunately cannot be corrected and left as Aphakia (unfavorable prognosis anticipated), see next figure for details and overall percentages.

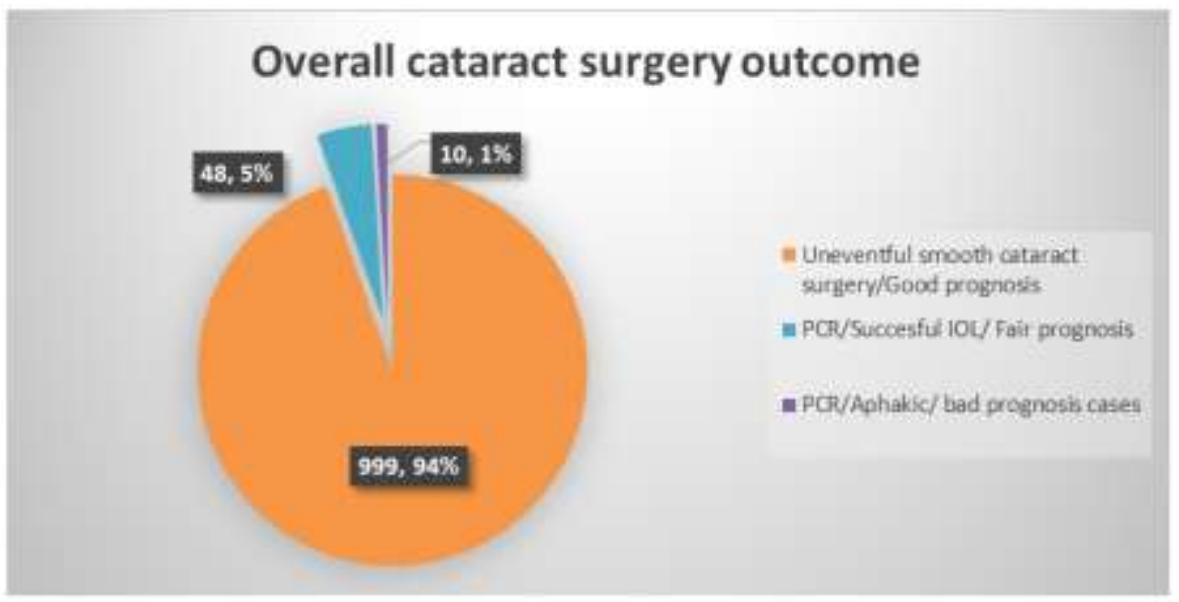

Fig-3: Pie chart of final refractive status as an indication to prognosis 
For the axial length measure, from 968 cases that taken, the mean was 23.21, median 23.14 and standard deviation was $1.0929,89$ cases were missing (Figure-2).

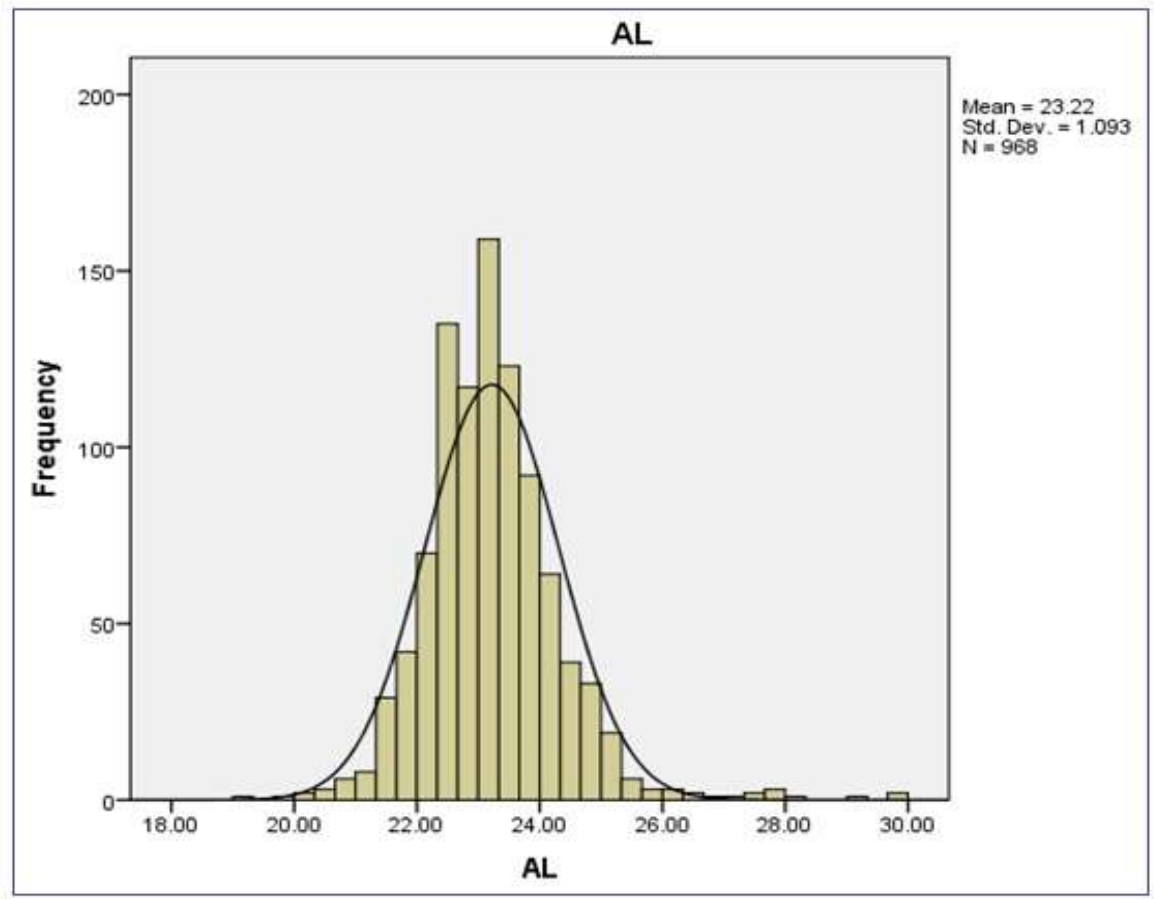

Fig-4: Histogram of axial length distribution

Table-1: Refractive errors according (AL) axial length 6]

\begin{tabular}{|l|l|}
\hline AL group & Axial length \\
\hline Hypermetric & $>22 \mathrm{~mm}$ \\
\hline Emmetropic & $22-24.5 \mathrm{~mm}$ \\
\hline myopic & $<24.5 \mathrm{~mm}$ \\
\hline
\end{tabular}

Regarding the laterality eye, 538 (50.9\%) eyes was right eye and $519(49.1 \%)$ eyes was left, the visual acuity range is $6 / 9$ - LP and there are missing data included children under age of 6 years, some old patients could not answer and cases of mentally retard also their VA was not performed and documented well, the reliability of the visual acuity data was considered, the reliable VA include those VA that performed at the time or one week before documenting information in the file. The reliable ones were 917 files $(86.7 \%)$.

In regard to the eye intraocular pressure (IOP), the mean was 13.49 , median 13.00 and standard deviation 4.546, the visual acuity and IOP had nonsignificant relation with the PCR consequently there P values are $(1.56,0.882)$ respectively.

History of hypertension and diabetes recorded because it is of important relationship to development of intraoperative complication [22], there are three cases of hypothyroidism, two cases of chronic renal failure, one case of lymphoma, two cases of morbid obesity, two cases of Down syndrome, one case of
Alzheimer disease, one case of mental retardation, three cases of cancer and numbers of patient with CVA and COAD were ignored because they did not have any PCR .Percentage of patients with no comorbidity were $39.6 \%$ (419 cases), hypertension was 40.3\% (426 cases), diabetes 5.5 (58 cases) and presence of both hypertension and diabetes was $14.4 \%$ (152 cases), other systemic association which are of importance are Hepatitis type B virus positive (HBV positive) cases which was six cases $(0.6 \%)$ and seven cases $(0.7 \%)$ who had allergy to drugs.

The percentages of smoking, non -smoker was $71.8 \%$ (759 cases), smoker was $9.9 \%$ (105 cases) and $\mathrm{x}$ smoker was $18.3 \%$ (193 cases), the general anesthesia were are $5.7 \%$ (60 cases) and local anesthesia were $94.2 \%$ (996 cases), type of anesthesia the local and general anesthesia had risk factor of $(5.5 \%, 5.4 \%$ respectively) and past medical history of hypertension, diabetes and both of them they have risk factor of $(5.2 \%$ and $5.9 \%$ respectively) but none of them have statistically significant risk for PCR.

The smoking had no significant relation for increasing the risk factor of PCR (95\% CI ( 0.43 to 2.50$)$ OR: 1.04) and P 0.91, the Axial length separated as a group to myopic, emmtropic and hypermetrope [6], the risk estimation and logistic regression done for each group, the result was not significant statistically Figure6. 
Table-2: 95\% CI, OR and P values of PMH, AL, type of anesthesia and smoking

\begin{tabular}{|l|l|l|l|l|}
\hline & $\begin{array}{l}\text { TOTAL NO. } \\
\text { N=1057 }\end{array}$ & $\begin{array}{l}\text { PCR NO. } \\
\text { N=58 }\end{array}$ & \multicolumn{2}{|l|}{ Simple logistic regression/chi-square } \\
\hline PMH & N\% & N\% & 95\% CI and OR & P values \\
\hline Hypertension & 94.8 & 5.2 & $0.89(0.52$ to 1.54) & 0.696 \\
\hline Diabetes & 94.8 & 5.2 & $0.93(0.28$ to 3.08) & 0.911 \\
\hline Hypertension+ diabetes & 94.1 & 5.9 & $1.09(0.52$ to 2.28) & 0.804 \\
\hline Type anesthesia & & & & \\
\hline LA & 94.5 & 5.5 & $1.11(0.33$ to 3.65) & 0.863 \\
\hline GA & 95.0 & 5.0 & $0.90(0.27$ to 2.96$)$ & 0.863 \\
\hline Smoking & & & & \\
\hline Non- smoker & 94.6 & 5.4 & $0.94(0.52-1.68)$ & 0.846 \\
\hline Smoker & 94.3 & 5.7 & $1.04(0.43-2.50)$ & 0.914 \\
\hline Ex- smoker & 94.3 & 5.7 & $1.05(0.53-2.06)$ & 0.886 \\
\hline Axial length & & & & 0.430 \\
\hline Myopic group & 96.7 & 3.3 & $0.622(0.190-2.044)$ & 0.933 \\
\hline Emmtropic group & 95.0 & 5.0 & $1.302(0.491-2.170)$ & 0.503 \\
\hline Hypermetropic group & 88.0 & 6.0 & $1.351(0.558-3.267)$ & \\
\hline
\end{tabular}

Other related ocular disease, there were 11 patients $(1.0 \%)$ had glaucoma, four patients $(0.4 \%)$ of congenital cataract, two patients $(0.25 \%)$ of traumatic cataract, three patients $(0.3 \%)$ of clear lens extraction, two patients $(0.2 \%)$ of combined operation (glaucoma and cataract) and two patients $(0.2 \%)$ of vitrectomy.

Pseudoexfoliation there were 55 eyes $(5.2 \%)$, but the degree of severity were not recorded.

The information about type of cataract gathered from either resident note or seniors admission paper, there were nucleus sclerosis of all grads $6.6 \%$, cortical cataract $1.9 \%$, posterior subcapsular $19.1 \%$, mature cataract 38.6,immature cataract $12.2 \%$, hypermature cataract $1.7 \%$, intumescent $0.9 \%$, brunescent cataract $1.7 \%$, post. Polar cataract $1.1 \%$, clear lens $0.3 \%$, aphakia $1.5 \%$, unknown cataract include those cases no specific type of cataract written there were $14.6 \%$ and four cases there were no information about type of cataract was written.

There was positive relationship between PCR and increasing in age, the $p$ value 0.003 it was significant (Figure-4).

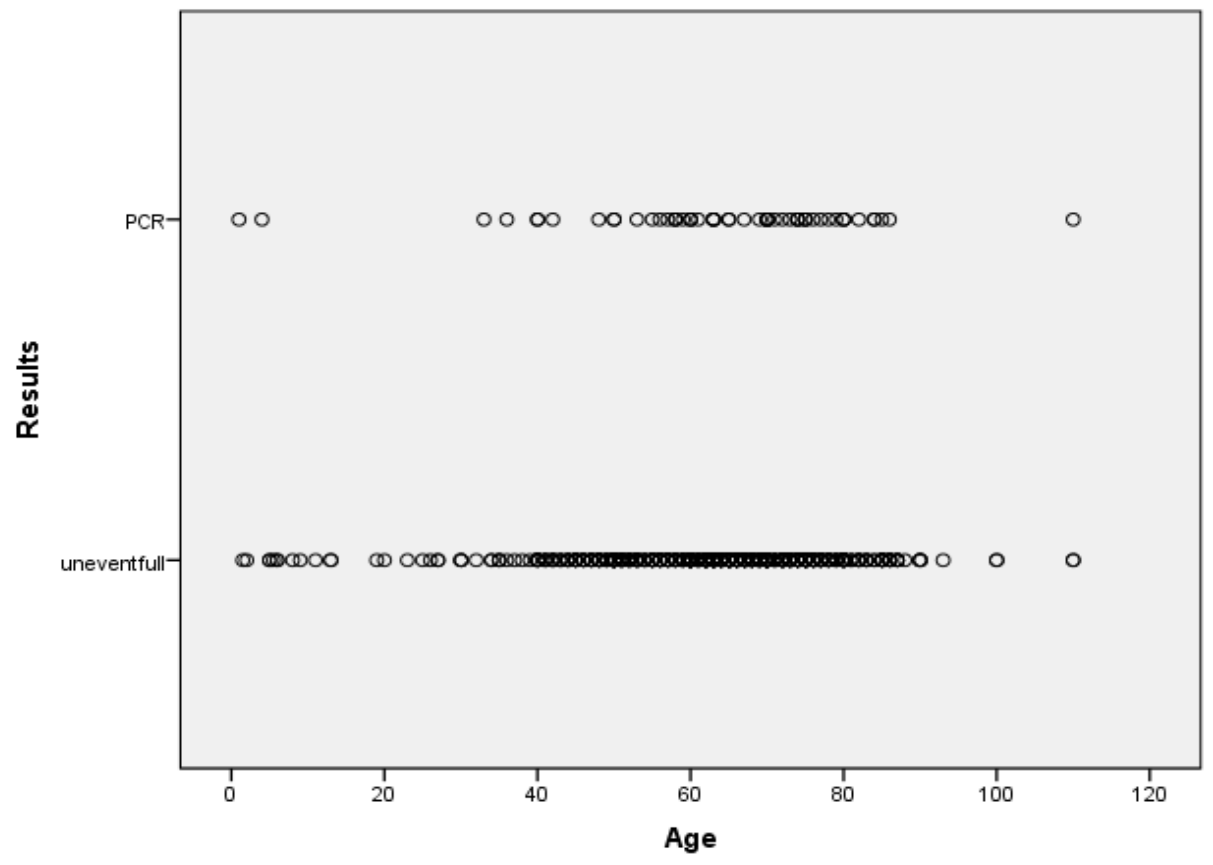

Error Bars: $95 \% \mathrm{Cl}$

Fig-5: A bar chart for relation of age and PCR 
Males to female risk of $(5.6 \%, 5.4 \%$ respectively), its statistically not significant risk for PCR, the laterality of eyes left eye had higher risk than right eye $(6.2 \%, 4.8 \%)$ but also not statistically significant risk for PCR.

Regarding the risk related to type of operation, of the 1075 eye underwent it, the ECCE risk factor of 6.9\% (95\% CI 1.03to3.13; OR: 1.80) P>0.036 for PCR, compared to the phacoemulsification and SICS had risk factor of $(4.0 \%, 2.9 \%$ respectively), type of cataract present in the conducted eyes, hypermature cataract had risk factor of $(22.2 \%)$, (95\% CI 1.17 to 16.98; OR: 5.40) and $\mathrm{p}>0.001$ for PCR compared to the other types which had no statistically significant risk for PCR, the risk factor of brunescent, mature and posterior capsular cataracts were $(11.1 \%, 5.4 \%$ and $5.4 \%$ respectively) but not significant risk for PCR the presence of Pseudoexfoliation and glaucoma did not increase the risk of PCR P value $0.227,0.00$ respectively, Table-3.

Table-3: 95\% CI, OR and P values of type of operation, type of cataract and PEX

\begin{tabular}{|l|l|l|l|l|}
\hline & $\begin{array}{l}\text { TOTAL NO. } \\
\text { N=1057 }\end{array}$ & $\begin{array}{l}\text { PCR NO. } \\
\text { N=58 }\end{array}$ & \multicolumn{2}{|l|}{ Simple logistic regression/chi-square } \\
\hline Type of operation & $\mathbf{N \%}$ & $\mathbf{N \%}$ & $\mathbf{9 5 \%}$ CI and OR & P Value \\
\hline Phacoemulsification & 96.0 & 4.0 & $0.59(0.33$ to 1.03$)$ & 0.063 \\
\hline ECCE & 93.1 & 6.9 & $1.80(1.03$ to 3.13$)$ & 0.036 \\
\hline SICS & 97.1 & 2.9 & $0.49(0.06$ to 3.70$)$ & 0.487 \\
\hline Type of cataract & & & & \\
\hline Hypermature & 77.8 & 22.2 & $540(1.71$ to 16.98$)$ & 0.001 \\
\hline Mature & 94.6 & 5.4 & $1.03(0.59$ to 1.79$)$ & 0.908 \\
\hline Brunescent & 88.9 & 11.1 & & \\
\hline PSC & 94.6 & 5.4 & & 0.227 \\
\hline PEX & 98.2 & 1.8 & $0.31(0.42$ to 2.29$)$ & \\
\hline NO PEX & 94.4 & 5.6 & & \\
\hline
\end{tabular}

\section{DISCUSSION}

The rate of $\mathrm{PCR}=5.5 \%$ in the patients who had cataract surgeries of over all 1057 eyes operated on by ophthalmologists with different surgical experience specialist surgeons to training residents, our result is lower in comparable with available data's about PCR by in the study done in Labbafinejad Medical Center in Iran by Zare $\mathrm{M}$ et al., [22] the rate of the PCR was $7.9 \%$ when cataract surgery done by fellows and residence and nearly the same in a study done by Tarbet et al., [23] reported a rate of 5.3\% among second-year residents, in other hand our result is higher in comparable with many available data's and information in text books , its $1.92 \%$ in a study done in UK [18] and it was $3.2 \%$ in study done at Malaysia [24].

The incidence of PCR in all literature reported different between 0.2 to $14 \%$ and vitreous loss between 1 and $5 \%$, this rate now decreased because of advanced technique and device, the incidence among the experienced surgeon, the PCR rate in these category at $0.45-3.6 \%$ when they do phacoemulsification and it increase to $4.8-11 \%$ when they are converting ECCE to phacoemulsification [11], The UK Cataract National Database of cataract surgery performed by all grades of surgeons reported a posterior capsular rupture rate of 1.92 per cent [16].

We can regard that as an indicator for prognosis in PCR patients as Good for those 19 eyes with primary PC-IOL, fair prognosis for 39 eyes with
AC-IOL and unfavorable prognosis for 10 eyes left Aphakic.

In addition to the finding the rate of PCR, we did the risk stratification according to our available data's in file system, in this study age is a significant risk factor for PCR correspond to other published studies increasing in age is one of the main risk factor, but the gender has no relation in this study, while in the others study, age and sex significantly influenced the incidence of vitreous loss such that it was more common among older female subject, like reported in a study done by Zare $\mathrm{M}$ et al., [22], while in a study done by Ergun ŞB et al male gender had more risk for developing PCR [19].

There is no consensus on the effect of systemic hypertension on the rate of vitreous loss. Several studies, as well as this study, have found no association, like in study done in a Malasiya [24]. But, Abbasoglu et al., [25] reported a 1.7-fold increase in the rate of vitreous loss with systemic hypertension.

Diabetes mellitus may indirectly influence the rate of posterior capsular rupture through different mechanisms including rigid pupil and previous pars plana vitrectomy. But in current study has no effect on the PCR p value was 0.9 , same result found in Zare M et al., [22] study the $\mathrm{p}$ value 0.7 no significant effect, while in a study reported in malyisa [24] $\mathrm{p}$ vale was < 0.001 , it has a strong association (Table-4). 
Pseudoexfoliation, it increased the risk of PCR through several mechanisms including zonular weakness and poor pupil dilatation [26] in the present study, it was found that there was no significant increase in the occurrence of posterior capsule rupture among patients with Pseudoexfoliation. As found in
THEVI Thanigasalam et al., [26], concluded that there was no correlation between the occurrence of posterior capsule rupture and the presence of Pseudoexfoliation among patients who underwent phacoemulsification, while in Abbasoglu et al., [25] reported a 2.5-fold risk of vitreous loss in eyes with Pseudoexfoliation.

Table-4: A comparison of $p$ value between our study and study done in Iran and Malaysia

\begin{tabular}{|l|l|l|l|}
\hline Risk factors & $\begin{array}{l}\text { In current study } \\
\text { P Values }\end{array}$ & $\begin{array}{l}\text { Zare M } \text { et al., } \\
\text { P Values }\end{array}$ & $\begin{array}{l}\text { Malaysia study } \\
\text { P Values }\end{array}$ \\
\hline Age & 0.003 & 0.02 & 0.001 \\
\hline Gender & 0.885 & 0.003 & 0.001 \\
\hline Laterality of eye & 0.341 & 0.2 & \\
\hline Hypertension\% & 0.696 & 0.23 & 0.056 \\
\hline Diabetes \% & 0.991 & 0.7 & $<0.001$ \\
\hline Pseudoexfoliation & 0.227 & 0.03 & 0.006 \\
\hline AL(myopia) & 0.430 & 0.04 & \\
\hline Type of anesthesia & 0.865 & 0.7 & \\
\hline
\end{tabular}

Table-5: A comparison of $95 \% \mathrm{CI}$ and $\mathrm{OR}$ with p values

\begin{tabular}{|l|l|l|l|l|}
\hline & $\mathbf{9 5 \%}$ CI and OR in current study & P Values & $\mathbf{9 5 \%}$ CI and OR Malaysia study & P Value \\
\hline Age & $8.74(0.78-97.88)$ & 0.003 & $1.23(1.08$ to 1.40$)$ & 0.001 \\
\hline Male & $1.05(0.61-1.78)$ & 0.885 & $1.11(1.04$ to 1.17$)$ & 0.001 \\
\hline Pseudoexfoliation & $0.31(0.42$ to 2.29$)$ & 0.227 & $1.43(1.12$ to 1.83$)$ & 0.006 \\
\hline Diabetes & $0.93(0.28$ to 3.08 & 0.991 & $1.19(1.12$ to 1.26$)$ & $<0.001$ \\
\hline Hypertension & $0.89(0.52$ to 1.54$)$ & 069 & $1.06(1.00$ to 1.13$)$ & 0.056 \\
\hline Phacoemulsification & $0.59(0.33$ to 1.03$)$ & 0.063 & & $<0.001$ \\
\hline ECCE & $1.80(1.03$ to 3.13$)$ & 0.036 & $1.31(1.22$ to 1.40$)$ & \\
\hline
\end{tabular}

Considering the type and severity of cataract, the highest rate of vitreous loss occurred in eyes with dense nuclear cataracts, in current study hyper mature had $22.2 \%$ risk rate followed by brunecence $11.1 \%$ and mature and posterior capsular cataract $5.4 \%$ which had same risk, while in a study done by Zare M et al., [22], the dense nuclear cataract had $(16.4 \%)$ followed by severe posterior subcapsular cataracts $(15.6 \%)$ and severe cortical cataracts $(14.5 \%)$.

The type of operation, in this study ECCE has a higher risk for PCR in contrast of a study done in Malaysia [24] in which the phacoemulsification had significant relation to increase rate of PCR.

From 1057 eyes, we have 58 eyes with PCR, $19(32.75 \%)$ eyes of them had posterior IOL, 13 eyes had AC IOL in same day and 16 eyes had secondary AC IOL, total $29(50 \%)$ eyes, while in Zare M et al., [22] study, among complicated 61 eyes from 767 eyes, posterior chamber IOL implantation was feasible in 54 $(88.5 \%)$ eyes, while 7 eyes required anterior chamber IOLs because of poor capsular support.

\section{Conclusions}

The rate of PCR in our hospital is less than nearby region and more than that found in literature, dealing with need to overcome this complication with better refractive correction, the age and type of cataract was a significant risk factor, the type of operation ECCE is significant it may be due to our specialist and residents more familiar to the ECCE than the phacoemulsification. Others like PEX, diabetes and glaucoma were non-significant risk factor may be due to inappropriate pre-operative assessment and inadequate information's pre and intraoperatively in the recording system.

\section{RECOMMENDATIONS}

It's better to take care with recording data system to be specific file for different ocular condition like for cataract and glaucoma, trauma, squint or other type of operation.

A new file system we introduced that suitable to be easily picked and uploaded to computerized recording system, that it cover different types of operation and with detailed information pre, intra and post operatively, its less time consuming, it will help the surgeons and residents for easier operations documentation and doing multiple study with less time and more informative.

Other surgical facilities must be available to deal with this serious complication like vitrectomy specialist with all necessary instruments and different types of IOL to deal immediately with the complicated cases. 


\section{LIMITATIONS}

Because of the main source of information regarding this work is the old files recording system, the registration of the necessary information regarding operation whether it is pre operation, intraoperative, is defective so it affect overall strength of the work and non-appropriate for risk stratification before operation.

\section{Conflict of interest: None.}

\section{Financial disclosure: None.}

Acknowledgement: Thanks to Shaheed Dr Aso Eye Hospital, computer department, Archiving department and the hospital manager, Dr Abdul Rahman Hama Amin Hussein.

\section{REFERENCES}

1. Bobrow, J. C., Beardsley, T. L., Jick, S. L., Rosenberg, L. F., Wiggins, M. N., \& Joseph, R. (2014). American Academy of Ophthalmology / lens and cataract. Section 11. San Francisco; (3,39-43,96-110,160-162).

2. Raman, M. (2008). Eye essentials cataract assessment, classification and management. China; chapter 5 techniques in cataract surgery, 90.

3. Asbell, P. A., Dualan, I., Mindel, J., Brocks, D., Ahmad, M., \& Epstein, S. (2005). Age-related cataract. The Lancet, 365(9459), 599-609.

4. WHO. (2000). Control of major blinding diseases and disorders. Fact sheet number 214. WHO, Geneva; (Available from http://www.who.int/ipcs/features/ehc_children.pdf (cited NOV. 10, 2018)

5. Fishkind, W. J. (2017). Phacoemulsification and Intraocular Lens Implantation: Mastering Techniques and Complications in Cataract Surgery. Thieme.

6. Khambhiphant, B., Sasiwilasagorn, S., Chatbunchachai, N., \& Pongpirul, K. (2016). Effect of pupillary dilation on Haigis formulacalculated intraocular lens power measurement by using optical biometry. Clinical Ophthalmology (Auckland, NZ), 10, 1405-1410.

7. WHO. (1997). Blindness and visual disability: socioeconomic aspects.Fact sheet number 145. WHO, Geneva; (Available from https://www.who.int/blindness/causes/en(Cited October. 30, 2018).

8. Grover, S., Fishman, G. A., Anderson, R. J., Tozatti, M. S., Heckenlively, J. R., Weleber, R. G., ... \& Brown Jr, J. (1999). Visual acuity impairment in patients with retinitis pigmentosa at age 45 years or older. Ophthalmology, 106(9), $1780-1785$.
9. Bowling, B. (2016). Kanskis clinical ophthalmology. $8^{\text {th }}$ ed. Edinburgh: Elsevier Saunders; (270-271, 281-289).

10. Speath, G. L., Danesh-Meyer, H. V., Ivan, G., \& Anseim, K. (2012). Ophthalmic surgery: Principle and Practise. 4th ed. chapter 6- clinical feature, diagnosis and differential diagnosis.

11. Arup, C. editor. (2017). Posterior capsular rent genesis and management [Internet]. India: springer nature; 2017 [cited 2018Nov19]. Available from: https://books.google.iq/books?id=4z42DgAAQBA $\mathrm{J} \&$ printsec $=$ frontcover $\# \mathrm{v}=$ onepage $\& \mathrm{q} \& \mathrm{f}=$ false incidence and significance of posterior capsular rupture (3-8), prophylaxis of the capsular rent (2325).

12. Chan, E., Mahroo, O. A., \& Spalton, D. J. (2010). Complications of cataract surgery. Clinical and Experimental Optometry, 93(6), 379-389.

13. Kelman, C. D. (1967). Phaco-emulsification and aspiration: a new technique of cataract removal: a preliminary report. American journal of ophthalmology, 64(1), 23-35.

14. Han, J. V., \& McGhee, C. N. (2018). When is a complication a complication in contemporary cataract surgery?. Clinical \& Experimental Ophthalmology, 46(1), 7-10.

15. Jaffe, N. S., Jaffe, M. S., \& Jaffe, G. F. (1997). Cataract surgery and its complications. $6^{\text {th }}$ edn. Mosby, St Louis.

16. Jaycock, P., Johnston, R. L., Taylor, H., Adams, M., Tole, D. M., \& Galloway, P. (2009). The Cataract National Dataset electronic multi-centre audit of 55,567 operations: updating benchmark standards of care in the United Kingdom and internationally. Eye (Lond); [cited 2018Nov19]. Available from: https://www.ncbi.nlm.nih.gov/pubmed/18034196

17. Ionides, A., Minassian, D., \& Tuft, S. (2001). Visual outcome following posterior capsule rupture during cataract surgery. British journal of ophthalmology, 85(2), 222-224.

18. Sparrow, J. M., Taylor, H., Qureshi, K., Smith, R., Birnie, K., \& Johnston, R. L. (2012). The Cataract National Dataset electronic multi-centre audit of 55567 operations: risk indicators for monocular visual acuity outcomes. Eye, 26(6), 821-826.

19. Ergun, Ş. B., Kocamış, S. İ., Çakmak, H. B., \& Çağıl, N. (2018). The evaluation of the risk factors for capsular complications in phacoemulsification. International ophthalmology, 38(5), 1851-1861.

20. Chang, D. F., Masket, S., Miller, K. M., BragaMele, R., Little, B. C., Mamalis, N., ... \& ASCRS Cataract Clinical Committee. (2009). Complications of sulcus placement of single-piece acrylic intraocular lenses: recommendations for backup IOL implantation following posterior capsule rupture. Journal of Cataract \& Refractive Surgery, 35(8), 1445-1458. 
21. Gogate PM. Small incision cataract surgery: Complications and mini-review. Indian journal of ophthalmology. 2009 Jan;57(1):45.

22. Zare, M., Javadi, M. A., Einollahi, B., BaradaranRafii, A. R., Feizi, S., \& Kiavash, V. (2009). Risk factors for posterior capsule rupture and vitreous loss during phacoemulsification. Journal of ophthalmic \& vision research, 4(4), 208.

23. Tarbet, K. J., Mamalis, N., Theurer, J., Jones, B. D., \& Olson, R. J. (1995). Complications and results of phacoemulsification performed by residents. Journal of Cataract \& Refractive Surgery, 21(6), 661-665.

24. Salowi, M. A., Chew, F. L., Adnan, T. H., King, C., Ismail, M., \& Goh, P. P. (2017). The
Malaysian Cataract Surgery Registry: risk indicators for posterior capsular rupture. British Journal of Ophthalmology, 101(11), 1466-1470.

25. Abbasoğlu, Ö. E., Hoşal, B., Tekeli, O., \& Gürsel, E. (2000). Risk factors for vitreous loss in cataract surgery. European journal ophthalmology, 10(3), 227-232.

26. Thanigasalam, T., Sahoo, S., \& Soe, H. H. K. (2014). Posterior Capsule Rupture during Phacoemulsification among Patients with Pseudoexfoliation-Is There A Correlation?. The Malaysian Journal of Medical Sciences: MJMS, 21(4), 51. 